\title{
Fibre-optic probe for fluorescence diagnostics with blood influence compensation
}

Evgeny Zherebtsov, Viktor Dremin, Elena Zharkikh, Angelina Zherebtsova, llya Rafailov, et al.

Evgeny Zherebtsov, Viktor Dremin, Elena Zharkikh, Angelina Zherebtsova, Ilya Rafailov, Andrey Dunaev, Natalia Bazieva, Edik Rafailov, "Fibre-optic probe for fluorescence diagnostics with blood influence compensation," Proc. SPIE 10493, Dynamics and Fluctuations in Biomedical Photonics XV, 104931L (26 February 2018); doi: 10.1117/12.2290431

SPIE. Event: SPIE BiOS, 2018, San Francisco, California, United States 


\title{
Fibre-optic probe for fluorescence diagnostics with blood influence compensation
}

\author{
Evgeny Zherebtsov*a, Viktor Dremin ${ }^{\mathrm{b}}$, Elena Zharkikh ${ }^{\mathrm{b}}$, Angelina Zherebtsova ${ }^{\mathrm{b}}$, \\ Ilya Rafailov ${ }^{\mathrm{c}}$, Andrey Dunaev ${ }^{\mathrm{b}}$, Natalia Bazieva ${ }^{\mathrm{a}}$, Edik Rafailov ${ }^{\mathrm{a}}$ \\ ${ }^{a}$ Aston Institute of Photonic Technologies, School of Engineering and Applied Science, Aston \\ University, Aston Triangle, Birmingham, UK B4 7ET; \\ ${ }^{b}$ Research and Development Center of Biomedical Photonics, Orel State University, 95 \\ Komsomolskaya St., Orel, Russia 302026; \\ ${ }^{\mathrm{c}}$ Aston Medical Technology Ltd, Birmingham, UK
}

\begin{abstract}
To minimise the influence of blood content on the fluorescence measurements in vivo, a fibre optical probe combining fluorescence and diffuse reflectance measurements was developed. For the inverse solution of the blood content recovery, a set of neural networks trained by the Monte Carlo generated learning set was used. An approach of fluorescence measurements triggered by simultaneous real-time measurements of blood content in living tissue during moderate changes in contact pressure of the optic probe is proposed. The method allows one to decrease the necessary pressure on the probe as well as increase the repeatability of the measurements. The developed approach was verified in a series of experiments on volunteers with fluorescence excitation at $365 \mathrm{~nm}$ and $450 \mathrm{~nm}$. The proposed technology is of particular interest in the development of new fluorescence-based optical biopsy systems.
\end{abstract}

Keywords: fluorescence measurements in vivo, attenuation correction, probe pressure, diffuse reflectance, blood content, neural network, Monte Carlo simulation

\section{INTRODUCTION}

Since the early 1990's, fluorescence measurements (FS) have become an object for wide research and industrial interest, especially in the field of life sciences. However, it is known that in vivo recorded fluorescence spectra are influenced by many factors. ${ }^{1}$ Often for the measurements, the most significant factor is an influence of blood absorption in the living tissue. ${ }^{2}$ The attenuation and changes of the fluorescence signal due to the blood volume alterations hide the changes linked with the concentration of fluorescent agents effectively. ${ }^{3}$ Moreover, the highly variable parameter directly affects the sampling depth and volume due to the absorption of the excitation radiation. At this time, several techniques have been developed to compensate for the influence of blood absorption on the fluorescence measurements in vivo. ${ }^{4}$ Most of them usually require additional optical measurements during the measurement of fluorescence intensity. ${ }^{5}$ In this case, to calculate the compensation factor, different types of models can be used. Primarily they are based on the analytical models or reverse Monte Carlo simulation. Nevertheless, most of them keep the inherent limits of the approaches, either lack of accuracy or relatively long computation time. Even though the study on the topic has been conducted for a sufficiently long time, the development of an effective and useful compensation method is still a task of great significance. ${ }^{4}$ It should be noted that in some cases an additional measuring channel of blood content parameters is able to significantly increase predictor strength of a diagnostic method to distinguish healthy and disease affected area. ${ }^{6,7,8}$

In this paper, we have analysed and tested an attenuation correction technique based on the moderate mechanical pressure on the optical probe. It is well known that the pressure applied on optical diagnostic probes is a significant factor affecting the results of measurements. ${ }^{9}$ One of the main causes of such effect is the influence of the pressure on local blood flow. On the other hand, the pressure is a well-known technique for controlling optical properties of biological tissue. It increases diagnostic depth and volume as well as allowing evaluation of actual values of optical parameters of biological tissue in vivo (parameters of absorption, scattering, polarisation, fluorescence ${ }^{13}$, etc.) by reducing the influence of blood absorption. ${ }^{10}$ The key point of the application of the technique in the clinical practice is repeatability of the measurements. Every measurement should be treated as an individual case because the same pressure applied to the living tissue of two different volunteers or even for the same person in different places will normally result in a different effect on blood volume fraction in the area under the probe. To tackle the problem in this work, we are offering an approach with

\footnotetext{
*e.zherebtsov@aston.ac.uk; phone +44 1212043703 ;www.aston.ac.uk/eas/research/groups/photonics
} 
measurements of blood content in the sampling volume of the probe as feedback. Having this information, it becomes possible to trigger on the fluorescent measurements when the pressure is enough to consider the blood absorption as insignificant.

To evaluate the blood volume fraction, a recently developed approach (A. Bykov, E. Zherebtsov, I. Meglinski, University of Oulu, 2016) combining neural network fitting based on the training set of modelled diffuse reflectance spectra obtained by Monte Carlo simulation has been employed. ${ }^{11}$ To implement this in practice a fibre optical probe combining fluorescence and diffuse reflectance measurements has been used.

Thus, this work aimed to develop and implement an approach for the blood content recovery as well as use the information for the compensation of the blood influence on the fluorescence measurements applicable for the real-time in vivo diagnostics. To this purpose, we experimentally estimate the potential of co-registering parameters of cutaneous blood content and the tissue fluorophore fluorescence.

\section{THE METHOD OF RESEARCH}

For a comprehensive diagnosis of the state of microcirculatory-tissue systems in real time and in vivo, it is currently promising to apply several diagnostic technologies simultaneously in one diagnostic volume. In that way, diffuse reflectance spectroscopy (DRS) can be used for correction of the data obtained during fluorescence studies (FS). To address the problem, a fibre optical probe combining fluorescence (2 emitting and 1 collecting fibres) and diffuse reflectance measurement (1 emitting and 1 collecting fibres) approaches has been used (Fig. 1). The probe was used for delivery of penetrating radiation and registration of back-reflected secondary radiation from the tissue. A $365 \mathrm{~nm}$ and $450 \mathrm{~nm}$ radiation sources were used for fluorescence excitation. The probing and collecting fibres have a diameter of 400 microns. The source-detector spacing for the FS channel is $1.5 \mathrm{~mm}$ whereas the distance for the DRS channel is $2 \mathrm{~mm}$. For safety reasons, as well as to keep photobleaching of the tissue at an acceptable level, probe radiation power of the $365 \mathrm{~nm}$ excitation wavelength at the output of the fibre probe did not exceed $1.5 \mathrm{~mW}$. To assess the safety of the probe, coupled with the LED, we used documentation of the International Commission On Non-Ionizing Radiation Protection (ICNIRP) ${ }^{12}$. The calculation gives the effective skin irradiance of $1.4 \mathrm{~W} / \mathrm{m}^{2}$. From the ICNIRP recommendations, the proposed protocol of using the probe was deemed to be safe. The output power for the $450 \mathrm{~nm}$ excitation wavelength did not exceed $3.5 \mathrm{~mW}$. The numerical aperture of the fibres was 0.22 .

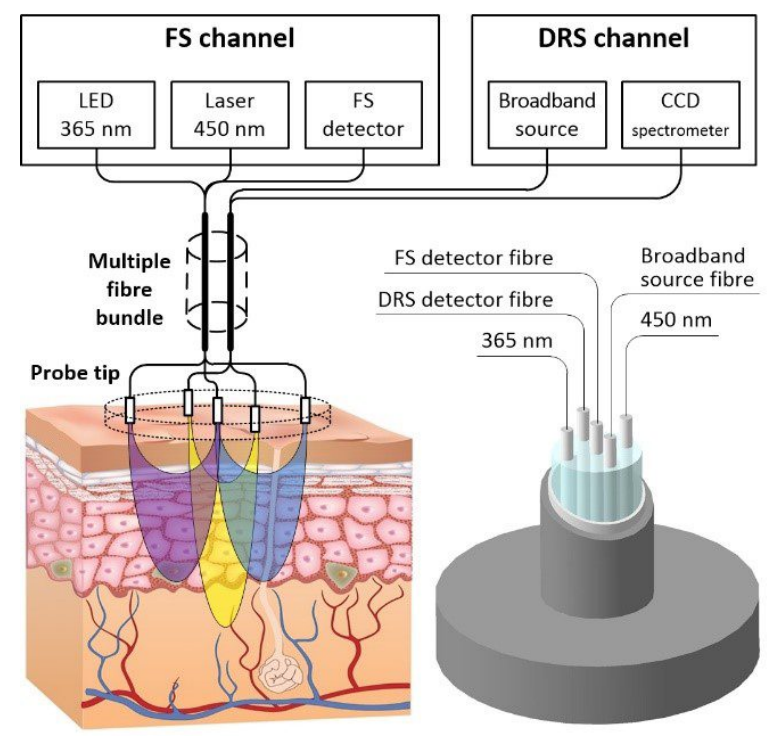

Figure 1. Measuring setup combines FS and diffuse reflectance measuring channels

To solve the inverse problem of estimation of skin chromophore content, an approach based on the neural network has been implemented (Fig. 2). A training set of spectra for the neural network was modelled by the Monte Carlo method. ${ }^{11} \mathrm{~A}$ CUDA-based Monte-Carlo simulation was used for routine calculation of diffuse reflectance spectra of skin, as well as 
their variations associated with possible physiological changes in the human skin. ${ }^{13}$ The differences in the spatial distribution of blood, melanin, oxygen saturation of blood, haematocrit, water content within the skin, as well as the numerical aperture and the angle of the detector positioning on the skin surface were taken into account. To model the diffuse reflectance spectra, a seven-layer model of skin was used. ${ }^{14}$ The values of the main variable parameters of the model are presented in Table 1. The parameters, which are the most significant factors affecting the skin reflectance, were varied to model all the possible sets of the values. In that way a training set of modelled diffuse reflectance spectra has been calculated for the different combinations of blood volume fraction, blood saturation, melanin fraction and epidermis thickness. The total training set had spectra in the range of 505-900 nm with a step of $5 \mathrm{~nm}$. Using the simulation data, the set of neural networks was trained to get the inverse solution for the blood content recovery.

Table 1. Variable parameters used for assessment of the absorption coefficients of the skin layers

\begin{tabular}{|l|c|c|c|}
\hline Parameter & Min value & Max value & Step \\
\hline Mean blood volume fraction $C_{\text {blood }} \%$ & 0 & 6 & 0.2 \\
\hline Blood saturation, $\%$ & 20 & 100 & 2 \\
\hline Melanin $C_{m e l}, \%$ & 0 & 6 & 1 \\
\hline Epidermis thickness, $\mu \mathrm{m}$ & 80 & 240 & 80 \\
\hline
\end{tabular}

Model of skin blood content measurements

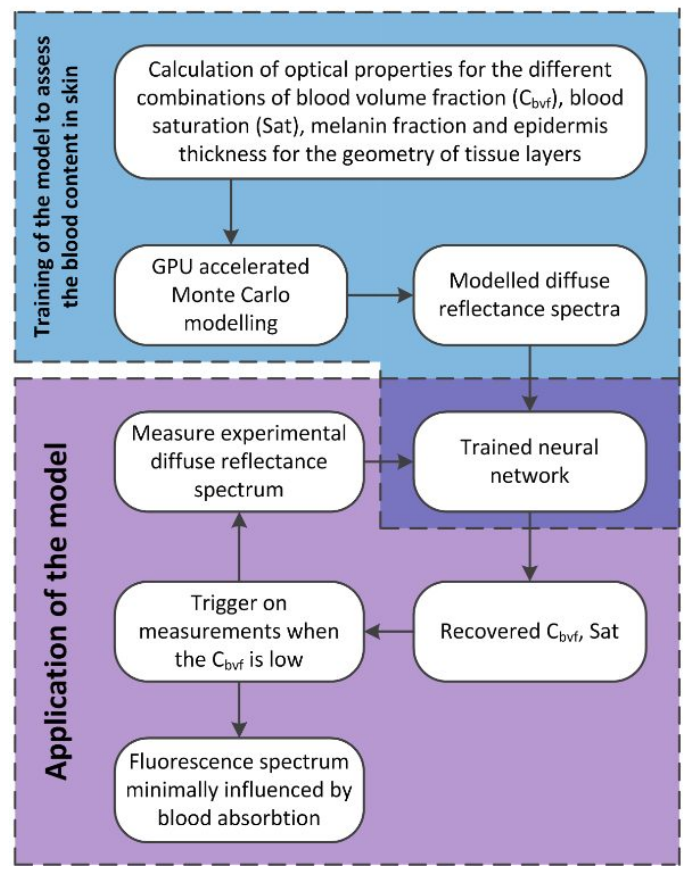

Figure 2. Flow chart of the approach for blood content estimation as well as minimisation of its influence at the registering fluorescence spectrum due to the moderate pressure applied to the probe

Usually, the probe pressure is one of the most prevalent factors which has a strong impact on the blood volume fraction in the area of probe placement. For the experimental validation of the approach, a series of experiments have been conducted in the skin palmar surface of the middle finger of 8 volunteers aged $24 \pm 7$ years with different pressure applied to the probe. The study was carried out in accordance with the principles outlined in the 2013 Declaration of Helsinki by the World Medical Association. The study protocol and its purpose were explained in detail to each subject and the informed consent was obtained from all of the subjects. To study the influence of local pressure on DRS and FS signals a special tooling set with calibrated weight, custom made by $3 \mathrm{D}$ printing, was used. The tooling was fixed on the optical probe, and the pressure value was changed by putting small weights in or taking them out of it. The influence of pressure on skin reflectance and 
intensity of skin fluorescence were registered at every step of the study. In that way, during each experiment, the pressure was changed stepwise from 0 to $36.2 \mathrm{mN} / \mathrm{mm}^{2}$.

\section{RESULTS (AND DISCUSSION)}

An example of the obtained experimental DRS and FS spectra with and without correction for different applied pressure to the probe (10 $\mathrm{mm}$ in diameter) is shown in Fig. 3.
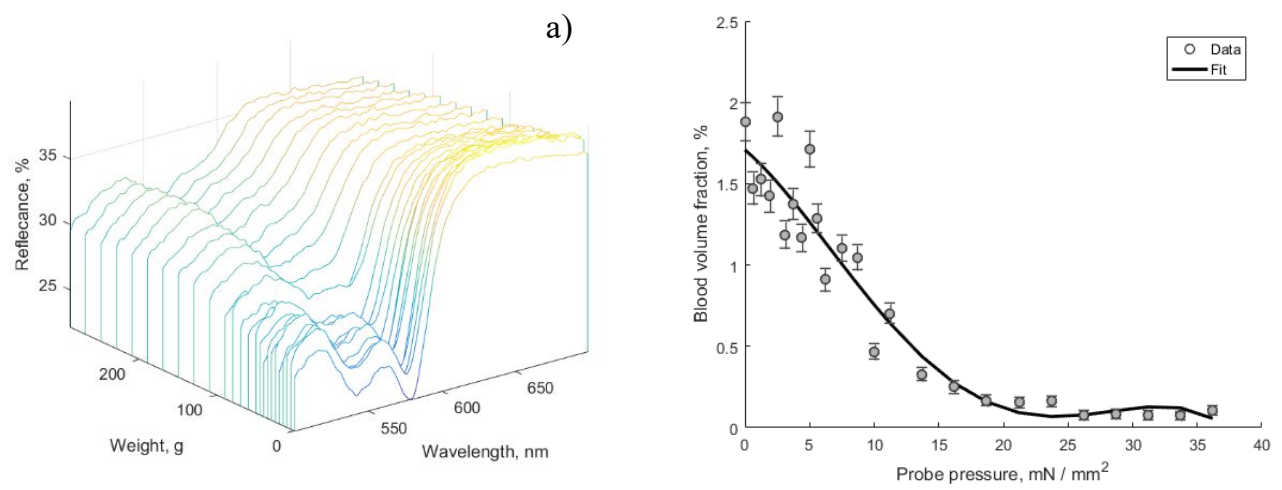

b)

c)

d)
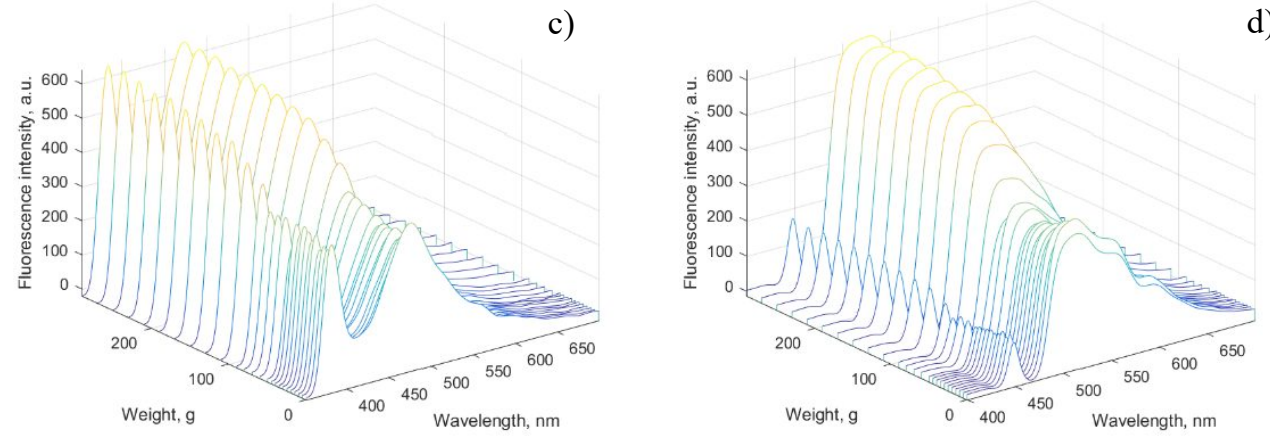

Figure 3. Experimental spectra for different loaded weights: a) DRS spectra; b) assessed blood volume fraction; c) FS spectra at UV $(365 \mathrm{~nm}) ;$ d) FS spectra at blue $(450 \mathrm{~nm})$

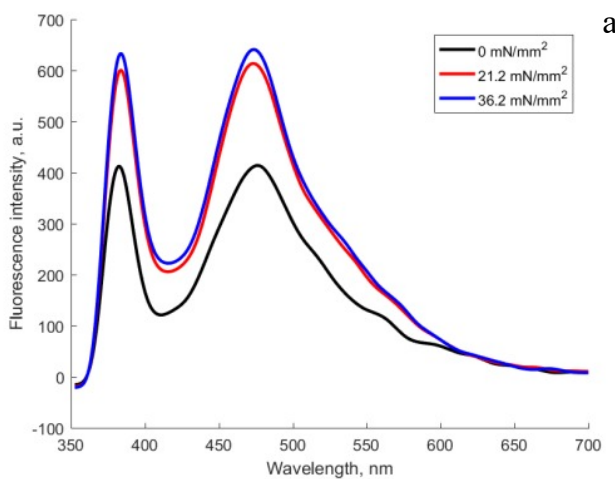

a)

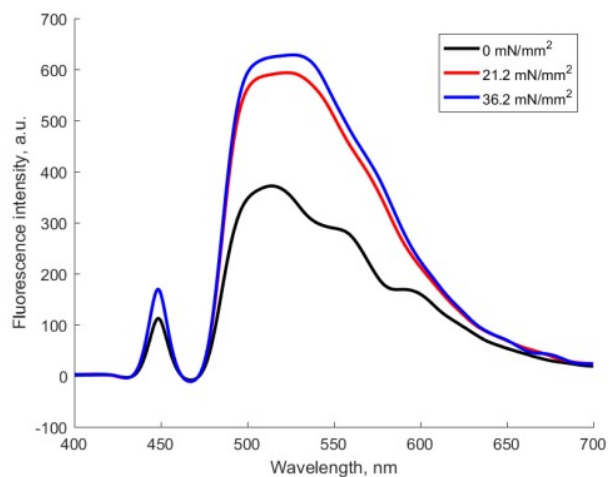

b)

Figure 4. Experimental fluorescence spectra for minimal and maximum probe pressure: a) FS spectra at UV $(365 \mathrm{~nm})$; b) FS spectra at blue $(450 \mathrm{~nm})$ 
The fluorescence spectra from the measurements with minimal $\left(\sim 0 \mathrm{mN} / \mathrm{mm}^{2}\right)$ and maximum $\left(36.2 \mathrm{mN} / \mathrm{mm}^{2}\right)$ pressure are shown in Fig. 4. In the same figure, the spectra selected on the basis of blood volume fraction measurements (pressure $21.2 \mathrm{mN} / \mathrm{mm}^{2}$, weight $170 \mathrm{~g}$ ) is shown. The data demonstrate that for the proposed blood volume fraction simultaneous measurements can serve as a trigger to launch the fluorescence measurements when the influence of the blood absorption can be supposed to be negligible. Furthermore, the actual information about the blood content in the sampling volume for every measured fluorescence spectrum makes it possible to accurately compensate the influence without pressure. As a development of the idea promising results have been obtained on the correction of fluorescence measurements based on the combination of recovery of the blood volume fraction by the proposed approach and lookup tables of the attenuation coefficients for skin model for certain wavelength of emission and blood content generated by the Monte Carlo modelling of fluorescence detection for the described probe.

\section{CONCLUSIONS}

The implemented multimodal approach for the in vivo optical measurements in combination with moderate pressure applied to the fibre optic probe for minimisation of blood influence has shown good repeatability of obtained experimental fluorescence spectra. The conducted experiment with different pressures applied to the probe has demonstrated that the proposed approach of fluorescence measurements triggered by simultaneous real-time measurements of blood content in living tissue allows one to decrease the required pressure on the probe as well as increase the repeatability of the measurements presented. The proposed model of blood content measurements combines both the speed of neural network processing and the accuracy and flexibility of Monte-Carlo modelling and can be used in real time measurements. The developed measurement technology is of particular interest in the development of new fluorescence-based optical biopsy systems.

\section{ACKNOWLEDGEMENTS}

The reported study has received funding from the European Union's Horizon 2020 research and innovation programme under the Marie Skłodowska-Curie grant agreement No. 703145. Authors also acknowledge funding from the Academy of Finland (grant 296408), RFBR according to the research project №18-02-00669 and grant of the President of the Russian Federation for state support of young Russian scientists № MK-3400.2018.8.

\section{REFERENCES}

[1] Dunaev, A. V., Dremin, V. V., Zherebtsov, E. A., Rafailov, I. E., Litvinova, K. S., Palmer, S. G., Stewart, N. A., Sokolovski, S. G. and Rafailov, E. U., "Individual variability analysis of fluorescence parameters measured in skin with different levels of nutritive blood flow," Med. Eng. Phys. 37(6) (2015).

[2] Lim, L., Nichols, B., Rajaram, N. and Tunnell, J. W., "Probe pressure effects on human skin diffuse reflectance and fluorescence spectroscopy measurements," J. Biomed. Opt. 16(1), 11012 (2011).

[3] Dremin, V. V, Zherebtsov, E. A., Rafailov, I. E., Vinokurov, A. Y., Novikova, I. N., Zherebtsova, A. I., Litvinova, K. S. and Dunaev, A. V., "The development of attenuation compensation models of fluorescence spectroscopy signals," 2016, 99170Y--99170Y--6.

[4] Bradley, R. S. and Thorniley, M. S., "A review of attenuation correction techniques for tissue fluorescence," J. R. Soc. Interface 3(6), 1-13 (2006).

[5] Georgakoudi I. Van Dam J., et al., J. B., "Fluorescence, reflectance and light scattering spectroscopy for evaluating dysplasia in patient with Barrett's esophagus.," Gastroenterology 120, 1620-1629 (2001).

[6] Dremin, V. V., Zherebtsov, E. A., Sidorov, V. V., Krupatkin, A. I., Makovik, I. N., Zherebtsova, A. I., Zharkikh, E. V., Potapova, E. V., Dunaev, A. V., Doronin, A. A., Bykov, A. V., Rafailov, I. E., Litvinova, K. S., Sokolovski, S. G. and Rafailov, E. U., "Multimodal optical measurement for study of lower limb tissue viability in patients with diabetes mellitus," J. Biomed. Opt. 22(8) (2017).

[7] Zherebtsov, E. A., Zherebtsova, A. I., Doronin, A., Dunaev, A. V., Podmasteryev, K. V., Bykov, A. and Meglinski, I., "Combined use of laser Doppler flowmetry and skin thermometry for functional diagnostics of intradermal finger vessels," J. Biomed. Opt. 22(4) (2017).

[8] Zharkikh, E. V., Dremin, V. V., Filina, M. A., Makovik, I. N., Potapova, E. V., Zherebtsov, E. A., Zherebtsova, A. I. and Dunaev, A. V., "Application of optical non-invasive methods to diagnose the state of the lower limb tissues in patients with diabetes mellitus," J. Phys. Conf. Ser. 929(1) (2017). 
[9] Zherebtsov, E. A., Kandurova, K. Y., Seryogina, E. S., Kozlov, I. O., Dremin, V. V., Zherebtsova, A. I., Dunaev,

A. V. and Meglinski, I., "The influence of local pressure on evaluation parameters of skin blood perfusion and fluorescence," 24 March 2017, 1033608, International Society for Optics and Photonics.

[10] Tuchin, V. V., [Tissue Optics: Light Scattering Methods and Instruments for Medical Diagnosis], Society of Photo-Optical Instrumentation Engineers (SPIE) (2015).

[11] Zherebtsov, E., Popov, A., Doronin, A., Meglinski, I. and Bykov, A., "Hyperspectral system for Imaging of skin chromophores and blood oxygenation,” Prog. Biomed. Opt. Imaging - Proc. SPIE 10412 (2017).

[12] "Guidelines on limits of exposure to ultraviolet radiation of wavelengths between $180 \mathrm{~nm}$ and $400 \mathrm{~nm}$ (incoherent optical radiation).”, Health Phys. 87(2), 171-186 (2004).

[13] Doronin, A. and Meglinski, I., "Online object oriented Monte Carlo computational tool for the needs of biomedical optics," Biomed. Opt. Express 2(9), 2461 (2011).

[14] Petrov, G. I., Doronin, A., Whelan, H. T., Meglinski, I. and Yakovlev, V. V., "Human tissue color as viewed in high dynamic range optical spectral transmission measurements.," Biomed. Opt. Express 3(9), 2154-2161 (2012). 